Textures and Microstructures, 1988, Vol. 10, pp. 59-65

Reprints available directly from the publisher

Photocopying permitted by license only

C) 1988 Gordon and Breach Science Publishers Inc.

Printed in the United Kingdom

\title{
An Assessment on Reliability of Quantitative Calculation Method for Obtaining Volume Fractions of Texture Components with the Reduced
ODF
}

\author{
L. ZUO, X. ZHAO, J. XU and Z. LIANG
}

Department of Materials Science and Engineering, Northeast University of Technology, Shenyang, China

(Received February 14, 1988)

The reliability and the accuracy of a quantitative calculation method for obtaining volume fractions of texture components of cubic materials were verified for the reduced ODFs terminated at $l \max =22$ and at $l \max =16$. It is concluded that an integrated width of $r= \pm 10^{\circ}$ around selected ideal orientations may be used in quantitative analysis of texture components. It is also shown that the conventional peak height method, which does not consider volume fractions, can only roughly give informations about texture components.

KEY WORDS: Complete ODF, Reduced ODF, Peak height values, Volume fractions.

\section{INTRODUCTION}

The true ODF describing the crystallite orientation distribution of a textured material in the form of series consists of the $l$-even terms and the $l$-odd terms. The ODF directly derived from experimental 
pole figures, restricted by Friedel's law, however, is only composed of the $l$-even terms (Matthies, 1979), namely the reduced ODF. Owing to the omission of the $l$-odd part, negative regions, ghosts and distorted orientation distributions appear in the reduced ODF space. Although several methods for recovering the true ODF have been proposed (Bunge and Esling, 1981a; Bunge and Esling, 1981b; Pospiech et al., 1981), yet they are not widely used for some reasons. So now only the reduced ODF is avaiable in practice.

In quantitative texture analysis, the volume fractions of materials in regions around ideal orientations are of interest. In order to confirm the reliability and the accuracy of quantitative analysis of texture components with the reduced ODF, a simulative investigation was carried out in the present paper, based on the method for determining the ODF by individual orientation measurements (Bunge, 1982; Liang et al., 1986). It is shown that an integration of the orientation distribution function about selected ideal orientations within $\pm 10^{\circ}$ may be suitable for quantitative determination of texture components with the reduced ODF

\section{PRINCIPLE}

For a given texture component with Miller indices $(h k l)$ [uvw], having orientation angles $\psi_{j}, \theta_{j}$ and $\varphi_{j}$ in the orientation space, one can obtain its volume fraction $f_{j}$ by integrating the orientation distribution function, $\omega(\theta, \psi, \varphi)$ (in Roe's notation), within $r^{0}$ of $\left\{\psi_{j}, \theta_{j}, \varphi_{j}\right\}$ (Flowers, 1983)

$$
f_{j}=k_{w} \cdot \iint_{\Delta \tau} \omega(\theta, \psi, \varphi) \cdot \sin \theta d \theta d \psi d \varphi
$$

where $\Delta \tau$ is the volume element formed around $\left\{\psi_{j}, \theta_{j}, \varphi_{j}\right\}$ within $r^{0}$ in the orientation space. The coefficient $k_{w}$ is 96 for cubic materials having orthogonal sample symmetries.

For a variety of preferred orientations, a simulation was made to determine the true ODF, the reduced ODF and volume fractions of texture components. In our work the orientation densities are within the limits of lower than 45 times in random units. The volume fraction around each ideal orientations, which was assumed 
to have a Gaussian distribution with a half-width value of $5^{\circ}, 10^{\circ}$ and $15^{\circ}$ respectively, was artificially given at first.

\section{RESULTS AND DISCUSSION}

The results show that in a sample consisting of a single texture component, as the number of the terms of the series expansion increases, the real peak positions in the reduced ODF do not change, but their peak heights get higher. If there are more than one texture component in a sample, the reduced ODF is a simple superposition of those of each component in the orientation space. Furthermore, the ODFs of the $l$-even part and the $l$-odd part are both maxima in the peak position of each components for multi and single texture component (see Figure 1). So the omission of the $l$-odd part makes that the peak heights of components in the reduced ODF are lower than those in the true ODF correspondently; the sharper a texture component, the greater the difference of the peak heights. These results are good agreement with the previous works (Matsuo et al., 1981; Jura et al., 1981).

A further simulation has found that the reduction of the peak heights caused by the omission of the $l$-odd part in the reduced ODF, compared with those in the true ODF of $l \max =22$, can reach about $30 \%$ for $l \max =22$ and about $60 \%$ or more for $l \max =16($ see Table 1 , Column 7, 8).

Since volume fractions of texture components are closely related to their peak positions, orientation distributions and scattering ranges in the orientation space, the conventional peak height method evaluating the amounts of components by the maximum values at their peak positions only has a qualitative meaning.

In order to examine the reliability and the accuracy of quantitative determination of volume fractions, a set of the orientation angles and the volume fractions of three components were used as input data of an ODF program based on individual orientation measurements and the true ODF of $l \max =22$, the reduced ODF of $l \max =22$ and $l \max =16$ were calculated respectively. One can find that the volume fraction of each component was computed with satisfactory fidelity in the condition of the same scattering width $\left(r= \pm 15^{\circ}\right)$ as the initial data for the three ODFs where the 


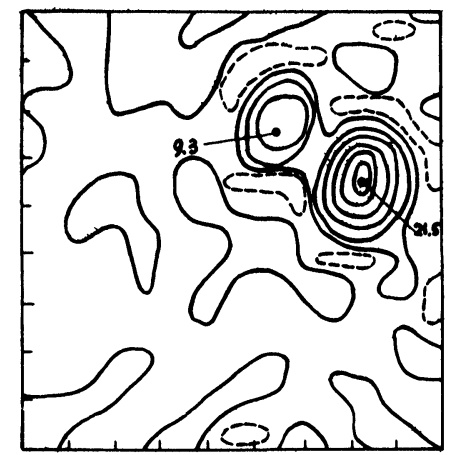

(a)

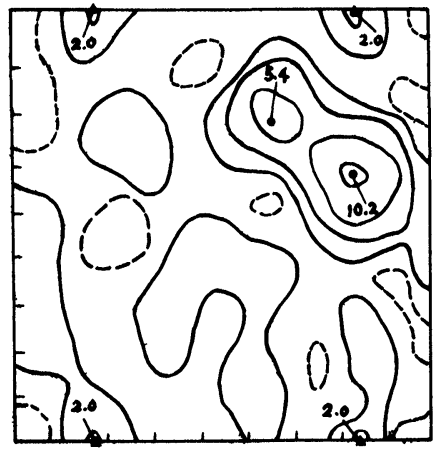

(c)

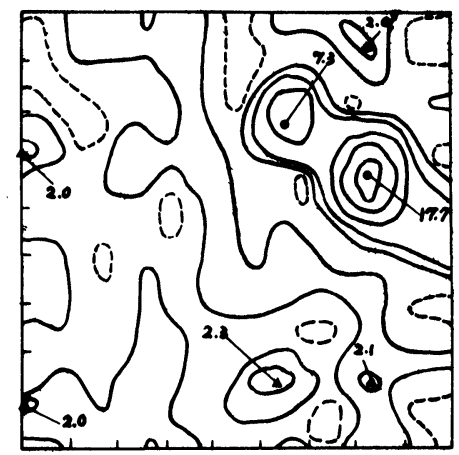

(b)

Intensity Ievel:

$0.1 .2,5,10,15.20$

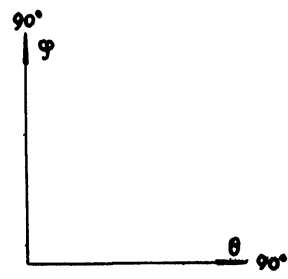

Figure 1 Crystallite orientation distribution function sections at $\psi=55^{\circ}$ for a single (231)[1̄11] component in Sample II. Contours are in times-random units. Dashed lines indicate negative regions. (a) the true ODF $(l \max =22)$; (b) the reduced ODF $(l \max =22)$; (c) the reduced ODF $(l \max =16)$. real peak positions, $\Delta$ ghost positions.

discrepancy of the reduced ODF of $l \max =22$ is slightly less than that of the reduced ODF of $l \max =16$ (see Table 1, Sample I). The values of volume fractions are also given in Table 1 for some other preferred orientations. If $r$ is taken as $\pm 10^{\circ}$, the relative discrepancies of volume fractions between the calculated values and the initial ones are lower than $20 \%$ for both the reduced ODFs at $l \max =22$ and at $l \max =16$; even in the condition that the peak position of one component is superimposed in the negative maximum position of another component in the orientation space (e.g., Table 1, Sample III), provided that the volume fraction of this 


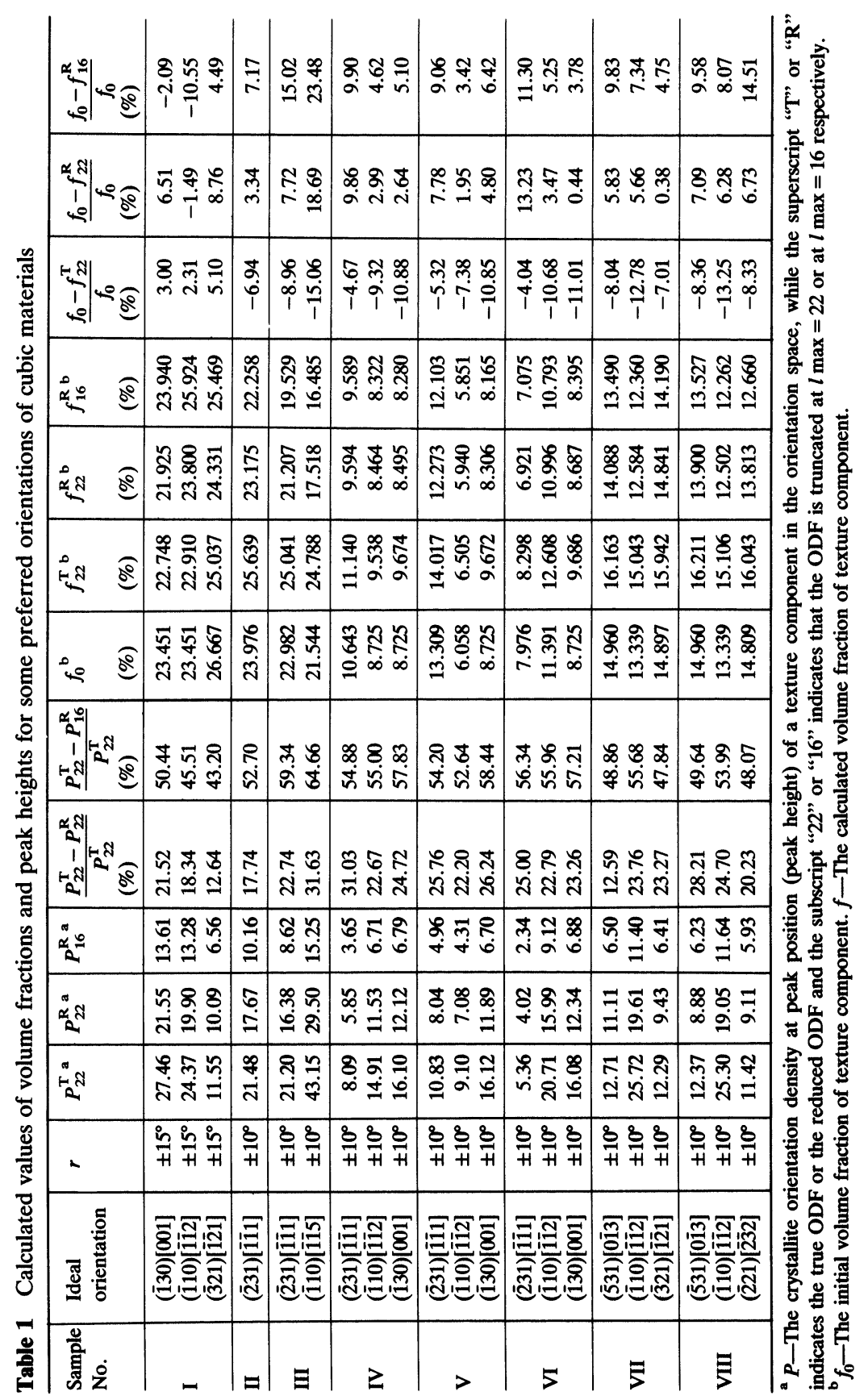


component is big enough, the fluctuation of volume fraction is about $20 \%$. (It is still in the range of experimental errors). So quantitative analysis of texture components may be satisfied with the reduced ODF of either $l \max =22$ or even $l \max =16$, if $r= \pm 10^{\circ}$ is taken.

For $r= \pm 5^{\circ}$, quite big discrepancy between the calculated volume fractions and the initial values were found. It is the fact that the omission of the $l$-odd part and the termination of the series lead to bad broadening and flattening of the texture component peak profiles which make the half-width values evidently larger than $5^{\circ}$ in the reduced ODF.

Since the error of the quantitative calculation for $r= \pm 5^{\circ}$ is too large and an integrated width of $r= \pm 15^{\circ}$ is too broad, a moderate width of $r= \pm 10^{\circ}$, which is tolerable for both the error and the scattering range, is recommended to be used in quantitative analysis of volume fractions.

\section{CONCLUSIONS}

The conventional peak height method evaluating the amounts of texture components by their peak heights is only qualitative. By using the quantitative calculation method of volume fractions, quantitative analysis of texture components may be carried out satisfactorily with only the experimental reduced ODF truncated at $l \max =22$ or even at $l \max =16$, which is widely used in the present texture analysis. An integrated width of $r= \pm 10^{\circ}$ around selected ideal orientations is recommended to be used in determining volume fractions of texture components of cubic materials.

\section{Acknowledgements}

This work is financially supported by the Natural Science Foundation of China, Grant 5870027.

\section{References}

Bunge, H. J. (1982). Texture Analysis in Materials Science (Morris, P. R., trans.), Butterworths, London, 50. 
Bunge, H. J. and Esling, C. (1981a). J. Appl. cryst., 14, 253.

Bunge, H. J. and Esling, C. (1981b). Proc. 6th Int. Conf. on Textures of Materials, ISIJ, Tokyo, Vol. 2, 1359.

Flowers, J. W. (1983). Textures and Microstructures, 5, 205.

Jura, J., Lücke, K. and Pospiech, J. (1981). Proc. 6th Int. Conf. on Textures of Materials, ISIJ, Tokyo, Vol. 2, 1402.

Matsuo, M., Tani, S. and Kawasaki, K. (1981). Proc. 6th Int. Conf. on Textures of Materials, ISIJ, Tokyo, Vol. 2, 1250.

Matthies, S. (1979). Phys. Stat. Sol. (b), 92, 135.

Liang Zhide, Xu Jiazheng and Wang Fu (1986). Zhigou Cailiao Sanweiquxiang Fenxishu-ODF Fenxi, NEUT Press, Shenyang, 115. 